October 1998

\title{
Weyl anomaly from Weyl gravity
}

\author{
SHIN'ICHI NOJIRI' and SERGEI D. ODINTSOV
}

Department of Mathematics and Physics

National Defence Academy, Hashirimizu Yokosuka 239, JAPAN

中 Tomsk Pedagogical University, 634041 Tomsk, RUSSIA

\begin{abstract}
We calculate the conformal anomaly from 5d Weyl gravity (with broken conformal symmetry) which is conjectured to be supergravity dual to $\mathcal{N}=2$ superconformal field theory via $\mathrm{AdS} / \mathrm{CFT}$ correspondence. Its comparison with $\mathcal{N}=2$ SCFT conformal anomaly (UV calculation) suggests that such duality may exist subject to presence of sub-leading $1 / N$ corrections to cosmological and gravitational constants.
\end{abstract}

\footnotetext{
${ }^{1}$ e-mail: nojiri@cc.nda.ac.jp, snojiri@yukawa.kyoto-u.ac.jp

${ }^{2}$ e-mail: odintsov@mail.tomsknet.ru, odintsov@itp.uni-leipzig.de
} 
For years, Weyl gravity attracts the attention as an alternative theory of gravity. There have been suggested mechanisms how to get usual general relativity (low energy behavior) from it, for example, via breaking of Weyl (or conformal) invariance.

Even if Weyl gravity itself is not realistic theory it can be important as an essential element of other models. An example of that sort is provided by string theory. According to refs. [1] there exists a scheme where the stringframe metric and dilaton dependent terms in low energy string action are given by

$$
\int d^{10} x \sqrt{G} \mathrm{e}^{-2 \phi}\left[R+4\left(\nabla_{\mu} \phi\right)^{2}+c_{1} W(R)\right] .
$$

Here $c_{1} \sim \alpha^{\prime 3}$ and $W(R) \sim C_{\mu \nu \alpha \beta}^{4}$. This is caused by the field redefinition ambiguity [2] which allows to change the coefficients of terms with Ricci tensor. In ref. [3] above action has been used to calculate strong coupling limit free energy for $\mathcal{N}=4$ super Yang-Mills theory via AdS/CFT correspondence (for an introduction, see [4]).

The usual form of Weyl gravity is given by the square of the Weyl tensor $C_{\mu \nu \rho \sigma} C^{\mu \nu \rho \sigma}$

$$
S=-\tilde{c} \int d^{5} x \sqrt{-g} C_{\mu \nu \rho \sigma} C^{\mu \nu \rho \sigma}
$$

In five dimensions,

$$
C_{\mu \nu \rho \sigma} C^{\mu \nu \rho \sigma}=\frac{1}{6} R^{2}-\frac{4}{3} R_{\mu \nu} R^{\mu \nu}+R_{\mu \nu \rho \sigma} R^{\mu \nu \rho \sigma} .
$$

Since Weyl tensor is, of course, invariant under the Weyl transformation:

$$
g_{\mu \nu} \rightarrow \mathrm{e}^{2 \sigma} g_{\mu \nu}
$$

the action (2) is Weyl invariant. The invariance is broken if we include the Einstein and cosmological terms:

$$
S=-\int d^{5} x \sqrt{-G}\left\{\frac{1}{\kappa^{2}} R-\Lambda+\tilde{c} C_{\mu \nu \rho \sigma} C^{\mu \nu \rho \sigma}\right\} .
$$

The string theory dual to $\mathcal{N}=2$ superconformal field theory is presumably IIB string on $\mathrm{AdS}_{5} \times X_{5}$ [5] where $X_{5}=S^{5} / Z_{2}$. (The $\mathcal{N}=2 S p(N)$ theory arises as the low-energy theory on the world volume on $N$ D3-branes sitting inside 8 D7-branes at an O7-brane). 
Then $\frac{1}{\kappa^{2}}$ and $\Lambda$ are given by

$$
\frac{1}{\kappa^{2}}=\frac{N^{2}}{4 \pi^{2}}, \quad \Lambda=-\frac{12 N^{2}}{4 \pi^{2}} .
$$

The Riemann curvature squared term in the above bulk action may be deduced from heterotic string via heterotic-type I duality [6]. Using field redefinition ambiguity [2] one can suppose that there exists the scheme where $R_{\mu \nu \alpha \beta}^{2}$ may be modified to $C_{\mu \nu \alpha \beta}^{2}$ in the same way as in ref. [1]. Then, the action (5) is presumably the bulk action dual to $\mathcal{N}=2 \mathrm{SCFT}$.

Our purpose here will be to check this conjecture, comparing the conformal anomaly in $S p(N) \mathcal{N}=2$ SCFT (UV calculation) and the conformal anomaly derived from the action (5) via AdS/CFT correspondence (SG side) [7].

Within AdS/CFT correspondence, the Weyl anomaly in 4 dimensions has been calculated from the 5 dimensional higher derivative action in [8] In the conjecture of AdS/CFT correspondence, the partition function in $d$ dimensional conformal field theory is given in terms of the classical action in $d+1$-dimensional gravity theory:

$$
Z_{d}\left(\phi_{0}\right)=\mathrm{e}^{-S_{\text {AdS }}\left(\phi^{\text {classical }}\left(\phi_{0}\right)\right)} .
$$

Here $\phi_{0}$ is the value of the field $\phi$ on the boundary and $\phi^{\text {classical }}\left(\phi_{0}\right)$ is a field on bulk background, which is AdS, given by solving the equations of motion with the boundary value $\phi_{0}$ on $M^{d} . S_{\mathrm{AdS}}\left(\phi^{\text {classical }}\left(\phi_{0}\right)\right)$ is the classical gravity action on AdS. When we substitute the classical solution into the action, the action, in general, contains infrared divergences coming from the infinite volume of AdS. Then we need to regularize the infrared divergence. It is known that as a result of the regularization and the renormalization there often appear anomalies. As fluctuations around the anti de Sitter space, we assume the metric has the following form:

$$
\begin{aligned}
d s^{2} & \equiv \hat{G}_{\mu \nu} d x^{\mu} d x^{\nu}=\frac{l^{2}}{4} \rho^{-2} d \rho d \rho+\sum_{i=1}^{d} \hat{g}_{i j} d x^{i} d x^{j} \\
\hat{g}_{i j} & =\rho^{-1} g_{i j} .
\end{aligned}
$$

We should note that there is a redundancy in the expression of (8). In fact, if we reparametrize the metric :

$$
\delta \rho=\delta \sigma \rho, \quad \delta g_{i j}=\delta \sigma g_{i j}
$$


by a constant parameter $\delta \sigma$, the expression (8) is invariant. The transformation (9) is nothing but the scale transformation on $M_{d}$. We expand the metric $g_{i j}$ as a power series with respect to $\rho$,

$$
g_{i j}=g_{(0) i j}+\rho g_{(1) i j}+\rho^{2} g_{(2) i j}+\cdots .
$$

We regard $g_{(0) i j}$ in (10) as independent field on $M_{d}$. We can solve $g_{(l) i j}$ $(l=1,2, \cdots)$ with respect to $g_{(0) i j}$ using equations of motion. When substituting the expression (10) into the classical action, the action diverges in general since the action contains the infinite volume integration on $M_{d+1}$. We regularize the infrared divergence by introducing a cutoff parameter $\epsilon$ :

$$
\int d^{d+1} x \rightarrow \int d^{d} x \int_{\epsilon} d \rho,\left.\quad \int_{M_{d}} d^{d} x(\cdots) \rightarrow \int d^{d} x(\cdots)\right|_{\rho=\epsilon} .
$$

Then the action can be expanded as power serie of $\epsilon$ :

$$
\begin{aligned}
S= & S_{0}\left(g_{(0) i j}\right) \epsilon^{-\frac{d}{2}}+S_{1}\left(g_{(0) i j}, g_{(1) i j}\right) \epsilon^{-\frac{d}{2}-1} \\
& +\cdots+S_{\ln } \ln \epsilon+S_{\frac{d}{2}}+\mathcal{O}\left(\epsilon^{\frac{1}{2}}\right)
\end{aligned}
$$

The term $S_{\ln }$ proportional to $\ln \epsilon$ appears when $d=$ even. In (12), the terms proportional to the inverse power of $\epsilon$ in the regularized action are invariant under the scale transformation

$$
\delta g_{(0) \mu \nu}=2 \delta \sigma g_{(0) \mu \nu}, \quad \delta \epsilon=2 \delta \sigma \epsilon .
$$

The invariance comes from the redanduncy (9). The subtraction of these terms proportional to the inverse power of $\epsilon$ does not break the invariance. When $d$ is even, however, there appears the term $S_{\ln }$ proportional to $\ln \epsilon$. The subtraction of the term $S_{\ln }$ breaks the invariance under the transformation(13). The reason is that the variation of the $\ln \epsilon$ term under the scale transformation (13) is finite when $\epsilon \rightarrow 0$ since $\ln \epsilon \rightarrow \ln \epsilon+\ln (2 \delta \sigma)$. Therefore the variation should be canceled by the variation of the finite term $S_{\frac{d}{2}}$ (which does not depend on $\epsilon$ )

$$
\delta S_{\frac{d}{2}}=-\ln (2 \sigma) S_{\ln }
$$

since the original total action $(\sqrt{16})$ is invariant under the scale transformation. Since the action $S_{\frac{d}{2}}$ can be regarded as the action renormalized by the 
subtraction of the terms which diverge when $\epsilon \rightarrow 0$, the $\ln \epsilon$ term $S_{\ln }$ gives the conformal anomaly $T$ of the renormalized theory on the boundary $M_{d}$ :

$$
S_{\ln }=-\frac{1}{2} \int d^{2} x \sqrt{-g_{(0)}} T
$$

With above procedure, for the general action

$$
\begin{aligned}
S= & \int d^{d+1} x \sqrt{-\hat{G}}\left\{a \hat{R}^{2}+b \hat{R}_{\mu \nu} \hat{R}^{\mu \nu}+c \hat{R}_{\mu \nu \rho \sigma} \hat{R}^{\mu \nu \rho \sigma}+\frac{1}{\kappa^{2}} \hat{R}-\Lambda\right\} \\
& +S_{B},
\end{aligned}
$$

we find the following anomaly for $d=4$ :

$$
T=\left(-\frac{l^{3}}{8 \kappa^{2}}+5 a l+b l\right)(G-F)+\frac{c l}{2}(G+F) .
$$

Here we introduced the length parameter $l$ by

$$
l^{2}=-\frac{\frac{12}{\kappa^{2}} \pm \sqrt{\frac{144}{\kappa^{4}}-4 d(d-3)\{(20 a+4 b+2 c\} \Lambda}}{2 \Lambda} .
$$

The sign in front of the root in the above equation may be chosen to be positive which corresponds to the Einstein gravity $(a=b=c=0)$. We also used the Gauss-Bonnet invariant $G$ and the square of the $4 \mathrm{~d}$ Weyl tensor $F$, which are given by

$$
\begin{aligned}
G & =R_{(0)}^{2}-4 R_{(0) i j} R_{(0)}^{i j}+R_{(0) i j k l} R_{(0)}^{i j k l} \\
F & =\frac{1}{3} R_{(0)}^{2}-2 R_{(0) i j} R_{(0)}^{i j}+R_{(0) i j k l} R_{(0)}^{i j k l} .
\end{aligned}
$$

Especially for the action (5), we find $\left(a=\frac{1}{6} \tilde{c}, b=-\frac{4}{3} \tilde{c}\right.$ and $\left.c=\tilde{c}\right)$

$$
T=\left(-\frac{l^{3}}{8 \kappa^{2}}-\frac{\tilde{c} l}{2}\right)(G-F)+\frac{\tilde{c} l}{2}(G+F) .
$$

We should note that $l$ is given by $l^{2}=-\frac{12}{\kappa^{2} \Lambda}$ from (18), especially in case of (6),

$$
l^{2}=-\frac{12}{\kappa^{2} \Lambda}=1
$$


For the $\mathcal{N}=2$ theory with the gauge group $S p(N)$, the usual UV Weyl anomaly is given by

$$
\begin{aligned}
T= & \frac{1}{24 \cdot 16 \pi^{2}}\left[-\left(8 N^{2}+6 N-\frac{1}{3}\right) R^{2}\right. \\
& \left.+\left(24 N^{2}+12 N\right) R_{i j} R^{i j}+(6 N-1) R_{i j k l} R^{i j k l}\right] \\
= & \frac{1}{24 \cdot 16 \pi^{2}}\left\{\left(-12 N^{2}-15 N+\frac{3}{2}\right)(G-F)+\left(3 N-\frac{1}{2}\right)(G+F)\right\} .
\end{aligned}
$$

Comparing (20) and (22), we find that the anomaly in $(20)$ can be reproduced if

$$
\tilde{c} l=\frac{6 N-1}{24 \cdot 16 \pi^{2}}, \quad \frac{l^{3}}{\kappa^{2}}=\frac{12 N^{2}+12 N-1}{3 \cdot 16 \pi^{2}} .
$$

The second equation in (23) is, however, not compatible with (6), where $\frac{l^{3}}{\kappa^{2}}=\frac{N^{2}}{4 \pi^{2}}$ since $l=1$ from (21). This might suggest that some sub-leading corrections to $\frac{1}{\kappa^{2}}$ and/or $\Lambda$ would be necessary. In other words, the bulk action (5) may be dual to $\mathcal{N}=2 S p(N)$ theory subject that some sub-leading corrections are induced to $\frac{1}{\kappa^{2}}$ (in minimal case) and to $\tilde{c}$. (One example of such sort is given by eqs.(23) with $l=1$ and cosmological constant equal to -12 of inverse gravitational constant.) The mechanism to produce such corrections is not yet clear. One possibility is that they come from field redefinitions.

In [9], the free energy of the corresponding $\mathcal{N}=2$ theory has been calculated from the Weyl gravity side (corresponding AdS black hole)

$$
F=-V_{3}\left(-\frac{\Lambda}{12}\right)^{-3} \frac{(\pi T)^{4}}{\kappa^{8}}\left(1-\frac{18 \tilde{c} \Lambda \kappa^{4}}{12}\right)
$$

By substituting (23) into (24) and fixing $l$ to be unity, we obtain

$$
F=-\frac{\pi^{2} V_{3} N^{2} T^{4}}{4}\left(1+\frac{17}{8 N}+\mathcal{O}\left(N^{-2}\right)\right) .
$$

On the other hand, from the field theoretical viewpoint (UV calculation of free energy in corresponding quantum field theory), we obtain

$$
F=-\frac{\pi^{2} V_{3} N^{2} T^{4}}{3}\left(1+\frac{2}{N}-\frac{1}{4 N^{2}}\right) .
$$


The coefficient $\frac{17}{8}$ of next-to-leading term in (25) is approximately 2 in the corresponding one in (26) besides the usual overall factor $\frac{4}{3}$. Such close correspondence indicates again that Weyl gravity action under discussion is indeed supergravity dual of super YM theory with two supersymmetries.

In case of $\mathcal{N}=4$ theory, the term proportional to $(G+F)$ does not appear in the Weyl anomaly. This requires $\tilde{c}=0$. Therefore the anomaly in $\mathcal{N}=4$ theory cannot be reproduced only by the squared Weyl tensor term.

More generally we can consider the $\mathcal{N}=2 S p(N)$ theory with $n_{V}=2 N^{2}+$ $N$ vectormultiplets and $n$ sets of $\left(n_{H}=\left(2 N^{2}+7 N-1\right) n\right.$ hypermultiplets

$$
\begin{aligned}
T= & \frac{1}{24 \cdot 16 \pi^{2}}\left[-\left(\frac{11 n_{V}}{3}+\frac{n_{H}}{3}\right) R^{2}\right. \\
& \left.+12 n_{H} R_{i j} R^{i j}+\left(n_{H}-n_{V}\right) R_{i j k l} R^{i j k l}\right] \\
= & \frac{1}{24 \cdot 16 \pi^{2}}\left\{\left(-\frac{9 n_{V}}{2}-\frac{3 n_{H}}{2}\right)(G-F)+\left(-\frac{n_{V}}{2}+\frac{n_{H}}{2}\right)(G+F)\right\} \\
= & \frac{1}{24 \cdot 16 \pi^{2}}\left\{\left(-\frac{9\left(2 N^{2}+N\right)}{2}-\frac{3\left(2 N^{2}+7 N-1\right) n}{2}\right)(G-F)\right. \\
& \left.+\left(-\frac{2 N^{2}+N}{2}+\frac{\left(2 N^{2}+7 N-1\right) n}{2}\right)(G+F)\right\} .
\end{aligned}
$$

By comparing (20) and (27) again, we find that the anomaly in (20) can be reproduced if

$$
\begin{aligned}
\tilde{c} l & =\frac{-n_{V}+n_{H}}{24 \cdot 16 \pi^{2}}=\frac{-2 N^{2}-N+\left(2 N^{2}+7 N-1\right) n}{24 \cdot 16 \pi^{2}} \\
\frac{l^{3}}{\kappa^{2}} & =\frac{5 n_{V}+n_{H}}{3 \cdot 16 \pi^{2}}=\frac{5\left(2 N^{2}+N\right)+\left(2 N^{2}+7 N-1\right) n}{3 \cdot 16 \pi^{2}} .
\end{aligned}
$$

We again come to the same conclusion: bulk action (5) may be dual to $\mathcal{N}=2$ SCFT subject to existance of sub-leading corrections to $\frac{1}{\kappa^{2}}$ and/or $\Lambda$. Another alternative could be the presence of some extra terms (fields) in action (5). That requires very careful investigation of string effective action.

Acknoweledgements. We thank A.Tseytlin for useful discussions.

\section{References}


[1] M.T. Grisaru and D. Zanon, Phys.Lett. B177 (1986) 347; M.D. Freeman, C.N. Pope, M.F. Sohnius and K.S. Stelle, Phys.Lett. B178 (1986) 199.

[2] D.J. Gross and E. Witten, Nucl.Phys. B277 (1986) 1; A.A. Tseytlin, Phys.Lett. B176 (1986) 92.

[3] S. Gubser, I. Klebanov and A. Tseytlin, Nucl.Phys. B534 (1998) 202, hep-th/9805156.

[4] O. Aharony, S. Gubser, J. Maldacena, H. Ooguri and Y. Oz, hepth/9905111.

[5] A. Fayyazuddin and M. Spalinski, Nucl.Phys. B535 (1998) 219, hepth/9805096; O. Aharony, A. Fayyazuddin and J.M. Maldacena, JHEP 9807 (1998) 013, hep-th/9806159.

[6] A.A. Tseytlin, Nucl.Phys. B467 (1996) 383.

[7] E. Witten, Adv.Theor.Math.Phys. 2 (1998) 253.

[8] S. Nojiri and S.D. Odintsov, hep-th/9903033.

[9] S. Nojiri and S.D. Odintsov, hep-th/9908065. 